Received: 7 June 2017

Accepted: 4 October 2017

Published online: 19 October 2017

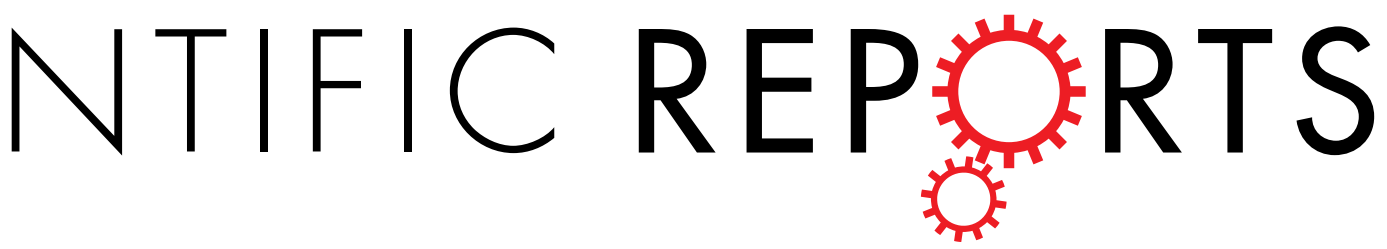

\title{
Comprehensive profiling of lysine ubiquitome reveals diverse functions of lysine ubiquitination in
} common wheat

\author{
Ning Zhang, Lingran Zhang, Chaonan Shi, Qiuzhen Tian, Guoguo Lv, Ying Wang, \\ Dangqun Cui \& Feng Chen
}

Protein ubiquitination, which is a major post-translational modifications that occurs in eukaryotic cells, is involved in diverse biological processes. To date, large-scale profiling of the ubiquitome in common wheat has not been reported, despite its status as the major cereal crop in the world. Here, we performed the first ubiquitome analysis of the common wheat (Triticum aestivum L.) variety, Aikang 58. Overall, 433 lysine modification sites were identified in 285 proteins in wheat seedlings, and four putative ubiquitination motifs were revealed. In particular, 83 of the 285 ubiquitinated proteins had ubiquitination orthologs in Oryza sativa L., and Arabidopsis thaliana. Ubiquitylated lysines were found to have a significantly different preference for secondary structures when compared with the all lysines. In accordance with previous studies, proteins related to binding and catalytic activity were predicted to be the preferential targets of lysine ubiquitination. Besides, protein interaction network analysis reveals that diverse interactions are modulated by protein ubiquitination. Bioinformatics analysis revealed that the ubiquitinated proteins were involved in diverse biological processes. Our data provides a global view of the ubiquitome in common wheat for the first time and lays a foundation for exploring the physiological role of lysine ubiquitination in wheat and other plants.

It is now abundantly clear that plant proteins are subjected to a wide array of post-translational modifications (PTMs) that greatly expand proteome functionality from more limited genomic information ${ }^{1}$. Among over 300 possibilities ${ }^{2}$, the majority of studies of PTMs on a proteomic scale in crops have focused on a small number of the most common types, such as phosphorylation, methylation, acetylation, glycosylation, ubiquitination, carbonylation and nitrosylation. Ubiquitin (Ub) is a protein composed of 76 amino acids found in both the cytosol and the nucleus of eukaryotic cells ${ }^{3,4}$. Protein degradation facilitated by the ubiquitin-proteasome system (UPS) is a major contributor to proteome remodeling ${ }^{5}$. In this pathway, ubiquitination occurs via the combination of Ub in lysine (Lys) residues of the acceptor proteins ${ }^{6}$. The process of ubiquitination typically leads to formation of an amide linkage comprising the $\varepsilon$-amine of lysine (Lys) of the target protein and the $\mathrm{C}$ terminus of $\mathrm{Ub}$ and can involve ubiquitination at distinct sites within the same protein ${ }^{7}$. This process occurs through a well-known enzymatic cascade involving E1 ubiquitin-activating enzymes, E2 ubiquitin-conjugating enzymes, and E3 ubiquitin ligases $^{4,6}$. Many studies have shown that ubiquitination also mediates many other cellular processes involved in growth and development of plants, such as embryogenesis, photomorphogenesis and hormone regulation, as well take part in immune/stress responses, membrane transport, DNA repair, chromatin remodeling, and transcriptional regulation ${ }^{2,4,8-10}$.

Proteome-wide approaches are desirable to obtain a global understanding of the role of ubiquitination. Hundreds or thousands of targets have been reported based on proteomic approaches via mass spectrometry (MS) analyses of yeast ${ }^{11}$ and mammalian cells ${ }^{12-14}$; however, only a relatively small number of ubiquitination sites were mapped although many ubiquitinated proteins were identified ${ }^{12}$, This is because the methods in these studies required enrichment of ubiquitinated proteins ${ }^{15}$. Accordingly, more robust methods of ubiquitylation site identification and quantification are needed. After the ubiquitinated proteins undergoes proteolytic digestion with

Agronomy College/National Key Laboratory of Wheat and Maize Crop Science/Collaborative Innovation Center of Henan Grain Crops, Henan Agricultural University, Zhengzhou, 450002, China. Correspondence and requests for materials should be addressed to F.C. (email: chf0088@163.com) 
trypsin, the diglycine (di-Gly) remnant derived from the two C-terminal glycine residues of ubiquitin remains covalently linked to the modified lysines. Thus, antibodies recognizing the di-Gly remnant on lysine residues enable the affinity capture of ubiquitinated peptides ${ }^{15}$. The di-Gly modification causes a mass shift (114.0429 Da) of the parent peptide, which enables identification of the precise location of ubiquitination sites based on peptide fragment masse ${ }^{15}$. Several proteome-wide in-depth ubiquitination analyses in mammalian cells were recently conducted based on di-Gly-Lys-specific antibody enrichment ${ }^{5,7,15}$, which led to identification of a large number of ubiquitination sites. In addition, the availability of this method has also been tested in plants (rice) ${ }^{16}$.

Common wheat (Triticum aestivum L.), also known as bread wheat, is one of the most important cereal crops in the world. In China, the Yellow and Huang wheat region is a major wheat-producing area, accounting for $60-70 \%$ of the national output. Because of its excellent drought resistance, disease resistance and resistance to frost, Aikang 58 has been the most popular cultivar in the Yellow and Huang wheat region for several years. Even though the ubiquitomes in Arabidopsis ${ }^{1,17-20}$ and rice (Oryza sativa $)^{16,21}$ have been studied, the proteome-wide ubiquitination data are still lacking for other plant species. To date, the proteome-wide identification of lysine-ubiquitinated proteins has not been accomplished in wheat. Elucidation of the ubiquitome in wheat cells is important for understanding the role of the UPS in regulating development and stress responses. In this study, we performed an overall profiling of the ubiquitome of leaves of two-leaf stage wheat seedlings using integrated proteomic techniques in which ubiquitylated peptides are directly enriched from a trypsin-digested whole wheat cell peptide mixture with a commercially high affinity anti-di-Gly-Lys-specific antibody ${ }^{7}$. We then analyzed lysine ubiquitination sites in wheat for the first time via highly sensitive MS and bioinformatics tools. A total of 433 lysine modification sites were identified on 285 proteins in wheat seedlings, controlling various biological processes such as signal transduction, transport, metabolism, and response to stimulus. These ubiquitinated proteins are localized in multiple compartments, mainly cell, membrane, and organelle. This study provides a global view of this important cereal crop ubiquitome and an abundant dataset for examination of functions of Ub-related proteins in wheat.

\section{Materials and Methods}

Plant material and growth conditions. The seeds of wheat cultivar Aikang58 were immersed and sterilized with $1 \%(\mathrm{w} / \mathrm{v}) \mathrm{H}_{2} \mathrm{O}_{2}$ for $0.5 \mathrm{~h}$, then were thoroughly washed with distilled water. The sterilized seeds were covered with water in Petri dishes for $24 \mathrm{~h}$ to germinate, after which they were grown in one-half strength Murashige and Skoog medium. The uniform seedlings $(\approx 4.1 \pm 0.03 \mathrm{~cm})$ were transferred into plastic pots with soil for growth. The wheat seedlings were kept in an illuminated incubator (RTOP-1000D, Zhejiang, China) at $25^{\circ} \mathrm{C} / 15^{\circ} \mathrm{C}$ day/night temperatures under a $16 \mathrm{~h} / 8 \mathrm{~h}$ light/dark photoperiod with $5500 \mathrm{Lx}$ light intensity and relative humidity of $70-75 \%$. The two-week-old seedlings with two fully expanded leaves from 10 single plants were then sampled, then frozen in liquid nitrogen rapidly. Finally, the treated samples were stored at $-80^{\circ} \mathrm{C}$ for protein extraction.

Protein extraction. Wheat leaves were ground in liquid nitrogen, after which five volumes of ice-cold $10 \%(\mathrm{w} / \mathrm{v})$ trichloroacetic acid (TCA) in acetone plus $0.07 \%(\mathrm{v} / \mathrm{v}) 2$-mercaptoethanol were added and samples were held at $-20^{\circ} \mathrm{C}$ for $4 \mathrm{~h}$. Next, the samples were centrifuged at $14,000 \mathrm{~g}$ for $30 \mathrm{~min}$ at $4{ }^{\circ} \mathrm{C}$, after which the supernatants were discarded and the pellets were washed three times with ice-cold acetone. The pellets were vacuum-dried and resuspended in lysis buffer ${ }^{22}$ using a Votex for $2 \mathrm{~h}$. The lysates were then sonicated at $80 \mathrm{~W}$ output with ten bursts of $10 \mathrm{~s}$ each, while being cooled on ice for $15 \mathrm{~s}$ between bursts, after which the suspension was centrifuged at $14,000 \mathrm{~g}$ for $40 \mathrm{~min}$ and $25^{\circ} \mathrm{C}$ to remove insoluble materials. The cleared lysates were collected and quantified by a Bradford assay (BioRad, California) based on a bovine serum albumin (BSA) standard ${ }^{23}$.

Tryptic digestion. A total of $10 \mathrm{mg}$ of protein were added to dithiothreitol (DTT) $(10 \mathrm{mM})$ and incubated for $2.5 \mathrm{~h}$ at $37^{\circ} \mathrm{C}$, after which they were cooled to room temperature, then alkylated with iodoacetamide (IAA) $(50 \mathrm{mM})$ for $30 \mathrm{~min}$ at room temperature in the darkness. The DTT and IAA-treated proteins were diluted by adding $100 \mathrm{mM} \mathrm{NH}_{4} \mathrm{HCO}_{3}$ to urea to give a final concentration $1.5 \mathrm{M}$, after which they were digested with trypsin (Promega) at a trypsin-to-protein ratio of 1:50 overnight $(18 \mathrm{~h})$. Finally, the resulting peptides were collected as a filtrate. After centrifugation for $20 \mathrm{~min}$ at $10,000 \mathrm{~g}$, the precipitates were removed and then the supernatants were desalted using Sep-Pak Classic C18 cartridges (Waters) followed by lyophilization.

Enrichment of ubiquitin-remnant-containing peptides. Lyophilized peptides were dissolved in immunoaffinity purification (IAP) buffer (50 mM MOPS- $\mathrm{NaOH}, \mathrm{pH} 7.2,10 \mathrm{mM} \mathrm{Na}_{2} \mathrm{HPO}_{4}$, and $50 \mathrm{mM} \mathrm{NaCl}$ ), then spun at $10,000 \mathrm{~g}$ at $4{ }^{\circ} \mathrm{C}$ for $10 \mathrm{~min}$. di-Gly-Lys antibody beads were used (PTMScan ubiquitin remnant motif K- $\varepsilon-G G$ kit, Cell Signaling Technology) and di-Gly-Lys-containing peptides were enriched as previously described $^{5}$.

Liquid chromatography (LC)-tandem mass spectroscopy (MS/MS) analysis. Enriched peptides were injected for LC-MS/MS analysis. The peptides were loaded onto a column (Thermo Scientific Acclaim PepMap 100, $100 \mu \mathrm{m} \times 2 \mathrm{~cm}$, nanoViper C18) connected to an analytical column (Thermo Scientific Easy Column, $10 \mathrm{~cm}$ long, $75 \mu \mathrm{m}$ inner diameter, $3 \mu \mathrm{m}$ resin) in buffer A ( $0.1 \%$ formic acid), then separated with a linear gradient of buffer $\mathrm{B}(84 \%$ acetonitrile and $0.1 \%$ formic acid) at a flow rate of $300 \mathrm{~nL} / \mathrm{min}$. The gradient was as follows (0 min-220 min, 0-55\% solvent B; $220 \mathrm{~min}-228 \mathrm{~min}, 55-100 \%$ solvent B; $228 \mathrm{~min}-240 \mathrm{~min}$, solvent B at $100 \%)$. LC-MS/MS analysis was conducted on a Q Exactive mass spectrometer (Thermo Scientific). The mass spectrometer was operated in positive ion mode. MS data was acquired using a data-dependent top 10 method dynamically choosing the most abundant precursor ions from the survey scan $(300-1800 \mathrm{~m} / \mathrm{z})$ for HCD fragmentation. Survey scans were acquired at a resolution of 70,000 at m/z 200 and resolution for HCD spectra was set to 17,500 at $\mathrm{m} / \mathrm{z} 200$. The normalized collision energy was $27 \mathrm{eV}$, and the underfill ratio, which specifies the 


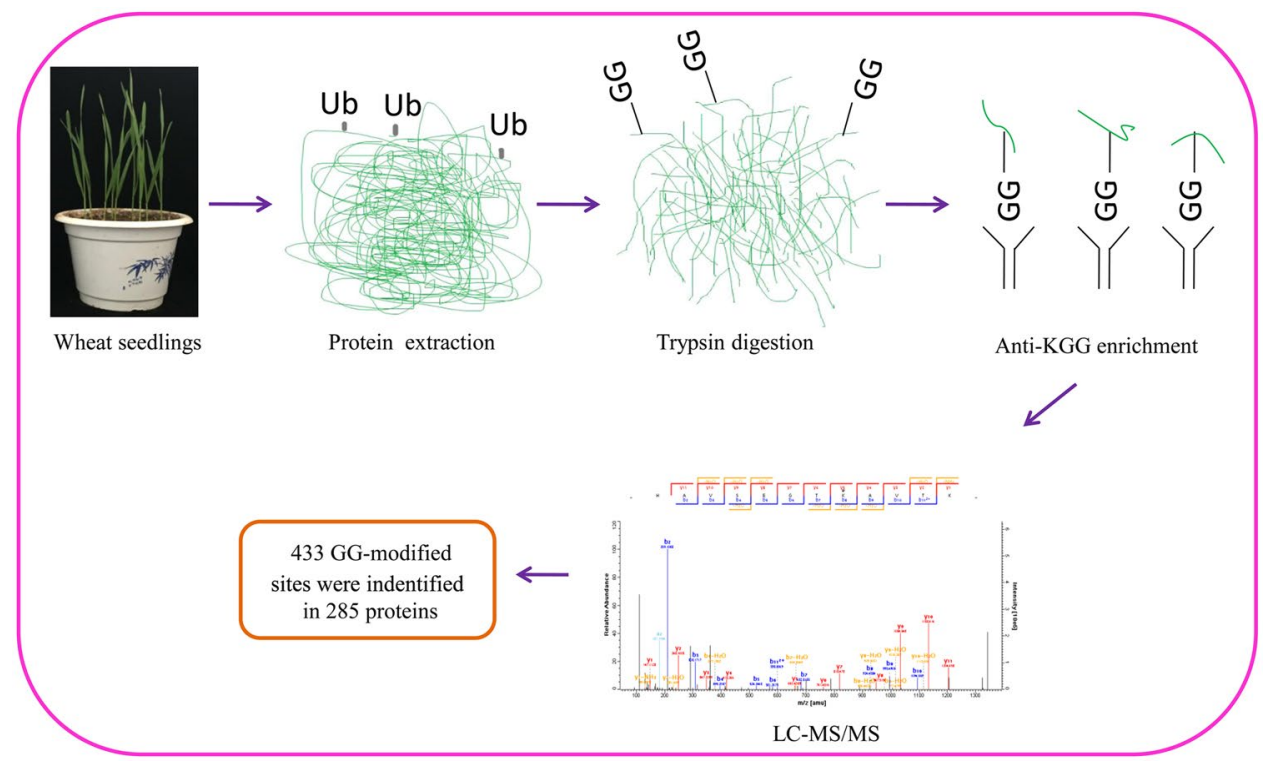

Figure 1. Overview of experimental procedures used in this study.

minimum percentage of the target value likely to be reached at the maximum fill time, was defined as $0.1 \%$. The mass spectrometry proteomics data have been deposited to the ProteomeXchange Consortium via the PRIDE ${ }^{24}$ partner repository with the dataset identifier PXD007243.

Data analysis. The MS data were analyzed using the MaxQuant software (version 1.3.0.5). MS data were searched against the Uniprot_Triticum_aestivum_101036_20160308.fasta (released at March 08, 2016, 101036 sequences). During the database search, the modifications were set as follows: main search ppm: 6; missed cleavage: 4; MS/MS tolerance ppm: 20; De-Isotopic: TRUE; enzyme: trypsin; fixed modification: carbamidomethyl (C); variable modification: oxidation (M), acetyl (Protein N-term), GlyGly (K); decoy database pattern: reverse; iBAQ: TRUE; match between runs: 2 min; minimum peptide length: 7; false discovery rate (FDR) thresholds for proteins, peptides and modification sites: 0.01 .

Bioinformatics analysis. The Motif-X software was used to analyze the model of sequences with amino acids in specific positions of ubiquityl-15-mers (seven amino acids upstream and downstream of the ubiquitination site) in all of the protein sequences ${ }^{16}$. The Arabidopsis thaliana proteome was used as the background database, the setting parameters were: occurrences $=20$, Bonferroni corrected $P$-value $=0.005$ (motif-x significance $=0.00018$ ), and the other parameters were set to the default values. Secondary structures of proteins were predicted by NetSurfP and $p$ value was calculated as previously described ${ }^{25}$. The Gene Ontology (GO) annotation proteome was derived from http://www.ebi.ac.uk/GOA and the lysine ubiquitination $\left(\mathrm{K}^{\mathrm{ub}}\right)$ proteins were classified by GO annotation based on three categories: biological processes, cellular components and molecular functions. The Kyoto Encyclopedia of Genes and Genomes (KEGG) database was used to annotate protein pathway ${ }^{26}$. WoLF PSORT, a subcellular localization predication program, was used to predict subcellular localization ${ }^{27}$. In addition, protein-protein interaction information of the surveyed proteins was retrieved from STRING software (http://string-db.org/). The results were downloaded as the tsv format and were imported into Cytoscape (version 3.2.1) software ${ }^{28}$ (http://www.cytoscape.org/) to visualize and further analyze functional protein-protein interaction networks. However, since Triticum aestivum does not appear to be in the STRING database, thus its high sequence similarity species barley (Hordeum vulgare L.) was used for STRING (protein with the similar sequence probably have the same function). Furthermore, BLASTP was conducted to evaluate the conservation of lysine-ubiquitinated proteins among wheat, rice and Arabidopsis thaliana according to previous report ${ }^{29}$.

\section{Results}

Proteome-wide analysis of lysine-ubiquitinated sites in wheat. An overview of experimental procedures used in this study is shown in Fig. 1. To identify lysine-ubiquitinated sites in wheat, proteins were extracted and digested into peptides with trypsin. Lysine-ubiquitinated peptides were then immune-enriched using a di-Gly-Lys-specific monoclonal antibody and analyzed by high-resolution LC-MS/MS. The mass errors of all identified peptides were checked and the results confirmed the high accuracy of the MS data (Fig. 2A). The length of the lysine-ubiquitinated peptides obtained was distributed between 7 and 31, which is in accordance with the property of tryptic peptides (Fig. 2B). Using this method, we identified 433 lysine-ubiquitinated sites in 285 unique proteins, which refer to 410 di-Gly-Lys-containing peptides (Additional file 1; Additional Fig. S1), and totals of 2505 non-modified peptides were identified (Additional file 2). To assess the distribution of ubiquitination sites in the lysine-ubiquitinated proteins of wheat, the numbers of identified modification sites per protein 

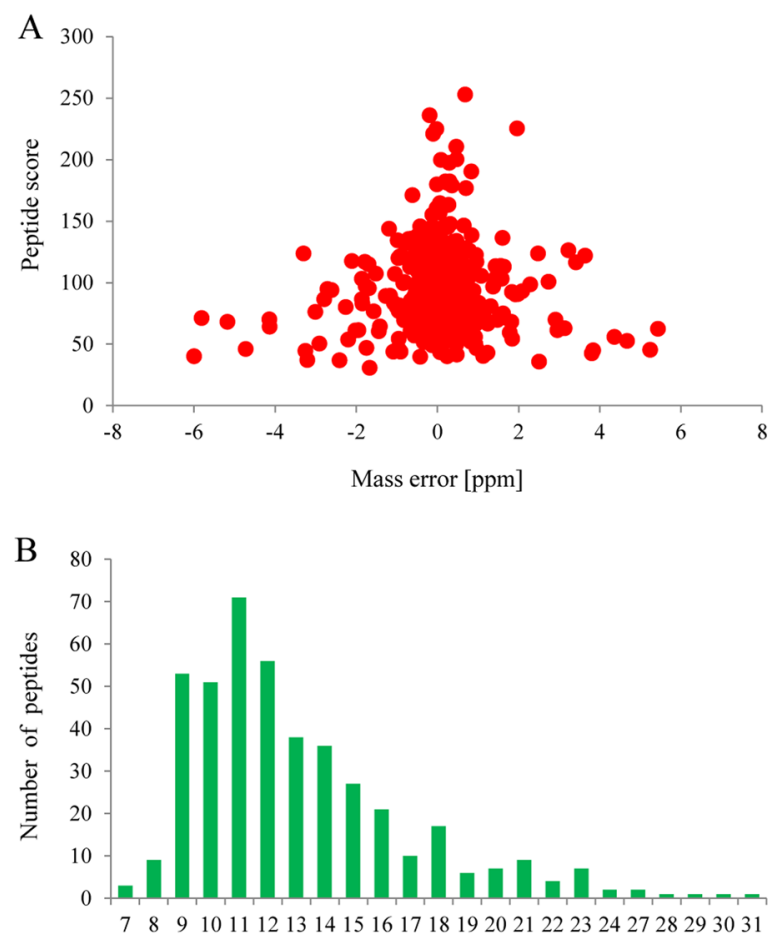

Peptide Length

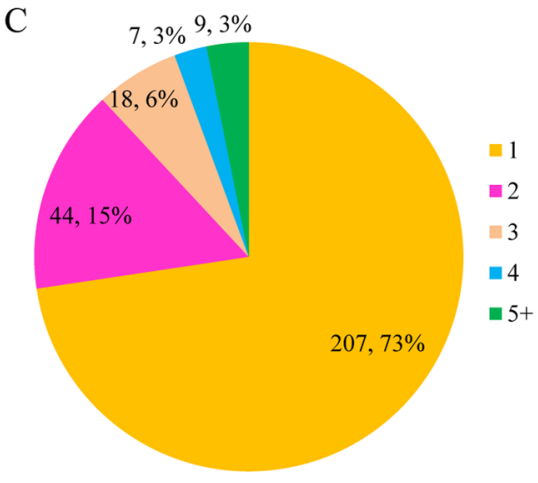

433 unbiquitinated sites in 285 proteins

Figure 2. Proteome-wide identification of lysine ubiquitination sites in wheat. (A) Mass error distribution of all identified peptides. (B) Peptide length distribution. (C) Pie chart illustrating the number and percentage of lysine ubiquitination sites per protein.

were calculated. The results indicated that $73 \%$ of proteins contained a single putative ubiquitination site, and the percentage of proteins with two, three, four and five or more modification sites were $13 \%, 5 \%, 4 \%$, and $5 \%$, respectively (Fig. 2C).

Trypsin proteolysis of proteins modified by ubiquitin, NEDD8, or ISG15 generates an identical di-Gly remnant on modified lysines, making it impossible to distinguish among these modifications by $\mathrm{MS}^{15}$. NEDD8-mediated modifications primarily target cullin subunits of cullin-RING E3 ubiquitin ligases in plants ${ }^{30}$, and the expression of ISG15 and its conjugation to lysines is relatively low in cells cultured under standard cell culture conditions $^{31}$. Thus, both NEDDylation and ISGylation are very rare when compared to ubiquitination ${ }^{32}$. Consequently, a majority of cellular peptides containing the di-Gly remnant stem from ubiquitylated proteins. Therefore, in this study, we refer to all di-Gly modified lysines as "ubiquitylation sites," even though a small fraction of these sites might be generated by modification of ISG15 or NEDD8.

Motif analysis of lysine ubiquitination sites. A previous study showed that conserved motifs might not exist in humans $s^{1,5,12}$, which is probably because ubiquitination sites lack the preference for specific amino acid residues at particular positions surrounding the ubiquitinated lysine in human cells. Or, this might be due to the use of an overly strict parameter setting during analysis by the Motif-X program. However, different organisms (e.g., mammals and plants) may have different sequence preferences in the ubiquitination sites. For instance, seven conserved motifs were identified in rice $^{16}$. To further determine the nature of the ubiquitinated lysines in wheat, we analyzed the context of the amino acid sequence surrounding the ubiquitinated lysines using the motif-x program. Of the 410 di-Gly-Lys-containing peptides, 398 had seven or more amino acids N- and C terminally surrounding the ubiquitinated lysine. Substantial bias in the amino acid distribution was observed from position -7 to +7 around the ubiquitinated lysines in the 398 peptides identified (Fig. 3A and Additional file 3). Four conserved sequences around the ubiquitination sites were found in the wheat ubiquitome; namely, $\mathrm{K}^{\mathrm{ub}} \mathrm{XA}$, $\mathrm{K}^{\mathrm{ub} X X A}$, AXXXXK $\mathrm{K}^{\mathrm{ub}}$, and $\mathrm{TXK}^{\mathrm{ub}}$ ( $\mathrm{K}^{\mathrm{ub}}$ indicates the ubiquitinated lysine, and $\mathrm{X}$ indicates any amino acid), which refer to $66,53,43$, and 28 unique peptides, respectively, and these exhibit different abundances, together accounting for about $47.7 \%$ of the ubiquitinated peptides identified (Fig. 3B). Moreover, analysis of the ubiquitinated lysine motifs showed that enrichment of one residue with a hydrophobic side chain, alanine (A), was observed at the $+2,+3$ or -5 position. Another type of amino acid with hydrophillic side chain, threonine $(\mathrm{T})$, was enriched in the -2 position (Fig. $3 \mathrm{~A}$ ). These results imply that amino acid residues with hydrophobic/hydrophillic side chains might be functionally important for ubiquitination on lysine residues of target proteins. It is noteworthy that $\mathrm{K}^{\mathrm{ub} X X A}$, one of these four ubiquitylation motifs, was also observed in rice which possibly implies that lysine ubiquitylation is a conserved PTM among different species. These three novel motifs in wheat would potentially provide an ubiquitination binding loci for future studies. 
A

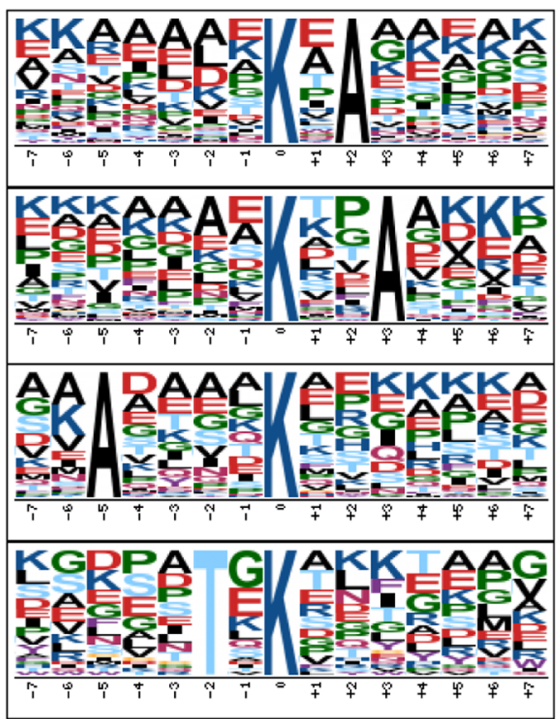

B

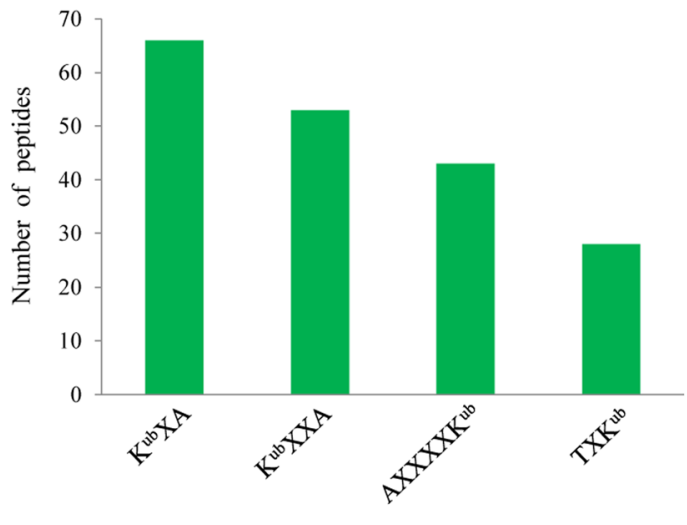

Unbiquitinated peptide motif

Figure 3. Ubiquitinated lysine motifs in wheat. (A) Ubiquitination motifs and the conservation of ubiquitination sites. The height of each letter corresponding to the frequency of the amino acid residue in that position. The central K stands for the ubiquitinated lysine. (B) The number of identified ubiquitinated peptides in each motif.

\section{A $\square$ All Lys $\quad$ Modified Lys}

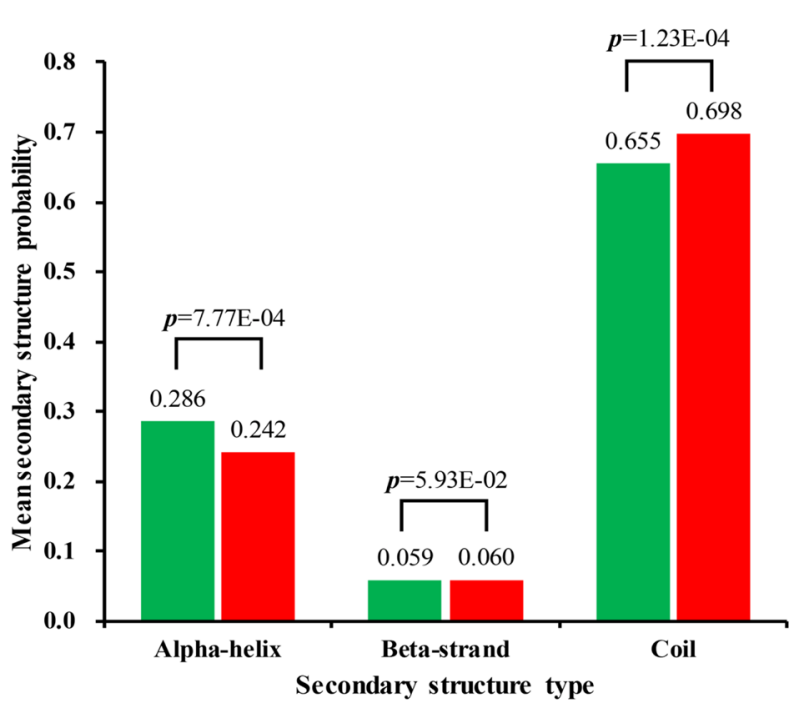

B

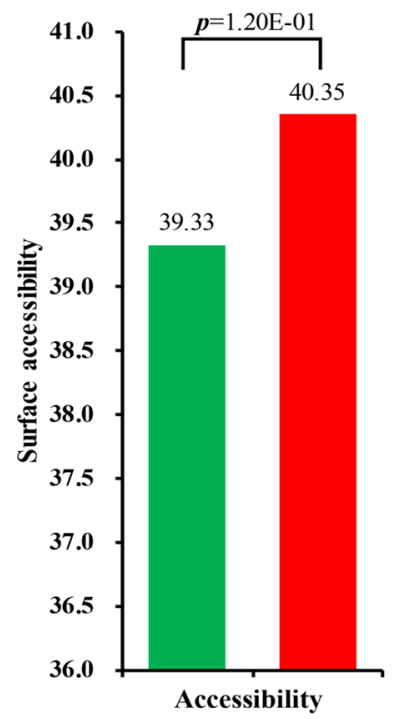

Figure 4. (A) Probabilities of lysine ubiquitination in different protein secondary structures (alpha-helix, betastrand and coli). (B) Predicted surface accessibility of ubiquitination sites. All lysine sites were in green and ubiquitinated lysine sites were in red.

To elucidate the properties of ubiquitylation sites, the local secondary structures of protein sequences surrounding ubiquitylation sites was investigated (Fig. 4A). The results indicated that $30.2 \%$ of the ubiquitination sites were located at regions with ordered secondary structures $(24.2 \%$ sites were located in the $\alpha$-helix and $6 \%$ were in the $\beta$-strand), while $69.8 \%$ of the ubiquitination sites were located in the disordered structures of proteins (coil). Moreover, ubiquitylated lysines have a significantly different preference for secondary structures when compared with all lysines. In general, ubiquitylated lysines are found in ordered $\alpha$-helix $(p=7.77 \mathrm{e}-04)$ and ( $\beta$-strand $p=5.93 \mathrm{e}-02)$ regions more frequently, while they are less frequent in unstructured coil regions $(p=1.23 \mathrm{e}-04)$. In addition to ordered regions, we further evaluated our identified lysine ubiquitylation sites for solvent accessibility. The results showed that $40.35 \%$ of the ubiquitinated lysine sites were exposed to the protein surface when compared with $39.33 \%$ of all lysine residues $(p=1.20 \mathrm{e}-01)$ (Fig. $4 \mathrm{~B})$. Therefore, the surface properties of proteins are not likely to be changed by lysine ubiquitination. 

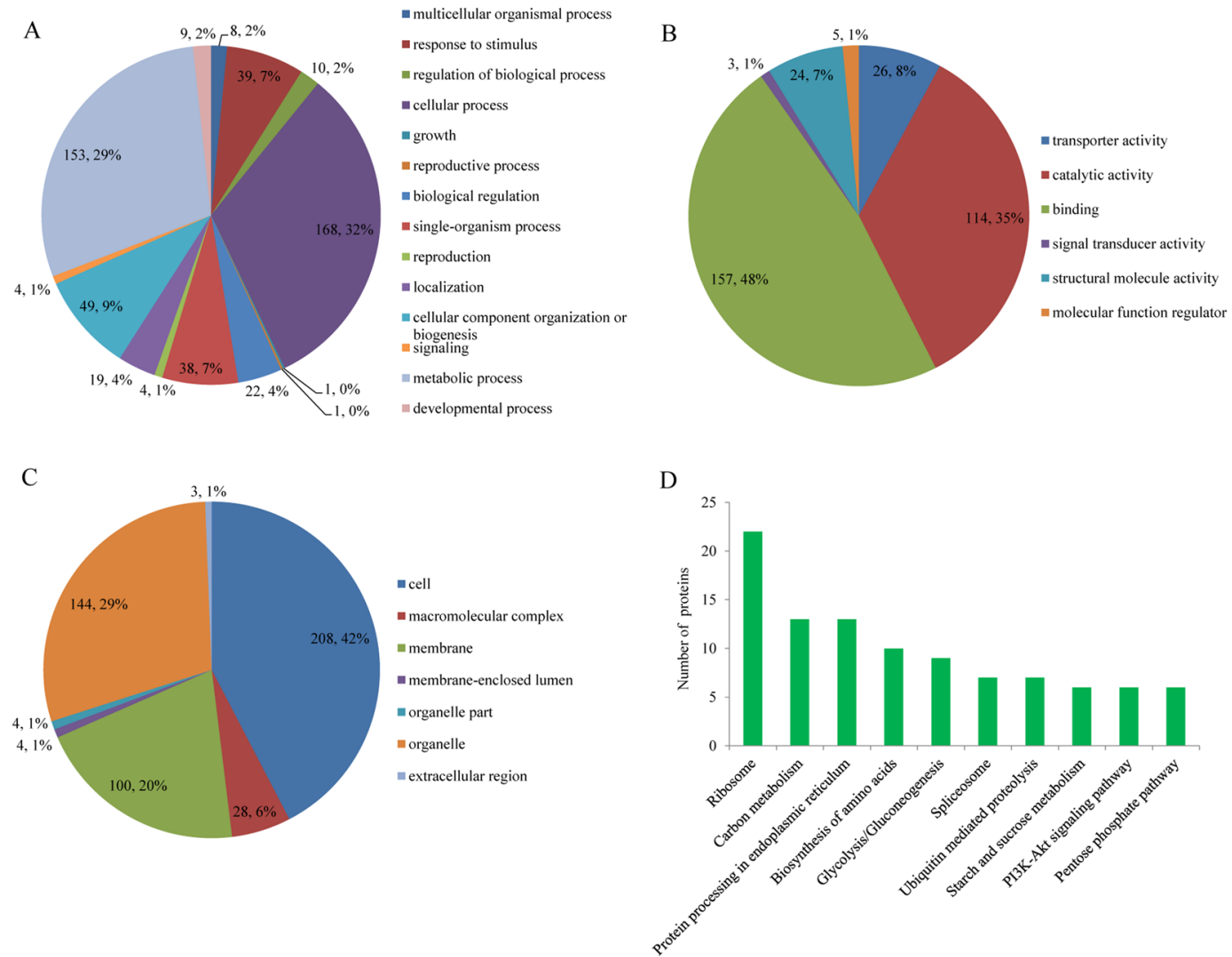

Figure 5. Pie charts of the distribution of ubiquitinated proteins based on their predicted molecular functions (A), biological processes (B), cellular components (C), and metabolic pathways (D).

Functional classification of ubiquitinated proteins. To better understand the ubiquitome in wheat, GO functional classification of the identified ubiquitinated proteins was conducted based on the biological processes, molecular functions, and cellular components (Fig. 5A-C and Additional file 4). Among the 285 ubiquitinated proteins grouped by their biological processes, some were found to be related to cellular process $(168,32 \%)$ and metabolic process $(153,29 \%)$, while others were assigned to responses to stimuli $(39,7 \%)$, and single-organism processes (38, 7\%) (Fig. 5A). Most ubiquitinated proteins in the molecular function classification were associated with binding activity $(157,48 \%)$ and catalytic activity $(114,35 \%)$ (Fig. 5B), suggesting that proteins may be involved in DNA transcription or protein interaction, and that enzymatic proteins are all subject to massive ubiquitination. Moreover, others were assigned to transporter activity $(26,8 \%)$ structural molecule activity $(24,7 \%)$, molecular function regulation (5, 1\%), and signal transducer activity $(3,1 \%)$. Subcellular localization analysis revealed that 208 (42\%) of the ubiquitinated proteins were located in cell, $144(29 \%)$ in organelle, and $100(20 \%)$ in the membrane (Fig. 5C). Furthermore, some proteins were predicted to be distributed in the macromolecular complex $(28,6 \%)$, membrane-enclosed lumen $(4,2 \%)$, organelle $(4,1 \%)$, and extracellular region $(3,1 \%)$ (Fig. 5C). The results of GO functional classification indicated that ubiquitinated proteins were involved in a broad range of biological processes and had various molecular functions in wheat. Below, we discuss the involvement of several biological processes in detail, specially notably enriched for proteins associated with proteasome composition, ribosome assembly/translation, membrane, carbohydrate metabolism, signal pathway, and photosynthesis (Table 1).

To gain insight into the ubiquitination mediated metabolic processes in wheat, we conducted KEGG pathway analysis. The results indicated that 285 ubiquitinated proteins were involved in 173 metabolic pathways (Fig. 5D and Additional file 5), primarily those associated with ribosome (22), carbon metabolism (13), protein processing in endoplasmic reticulum (13), biosynthesis of amino acids (10), and glycolysis/gluconeogenesis (9) (top 5, plant category). For example, in this study, a large set of 22 subunits of the $40 \mathrm{~S}$ and $60 \mathrm{~S}$ ribosome complexes ubiquitylated in wheat were identified (Fig. 6). During photosynthesis, the Calvin cycle converts carbon dioxide and other compounds into glucose. Several metabolic enzymes involved in the Calvin cycle were found to be ubiquitinated in wheat, including three isozymes of fructose-bisphosphate aldolase (W5HZ47, C1J959 and W5HA05), one ribulose 1,5-bisphosphate carboxylase/oxygenase large subunit (Rubisco) (W5BG62), one cytosolic putative fructose-1,6-bisphosphatase (D8L9K9), and one glyceraldehyde-3-phosphate dehydrogenase (A0A096UTL2) (Fig. 7).

Protein interaction network of ubiquitinated proteins in wheat. To further understand cellular processes regulated by ubiquitination in wheat, the protein interaction network was established (Fig. 8 and Additional file 6). Ubiquitylated proteins were grouped using associated GO biological process terms. The results 


\begin{tabular}{|c|c|c|c|}
\hline Accession no. ${ }^{\mathrm{a}}$ & Proteins & Accession no. ${ }^{\mathrm{a}}$ & Proteins \\
\hline $26 \mathrm{~S}$ proteasome & & $\begin{array}{l}\text { Carbohydrate } \\
\text { metabolism }\end{array}$ & \\
\hline W5BUE8 & $\begin{array}{l}\text { 26S proteasome non-atpase regulatory } \\
\text { subunit } 1\end{array}$ & W5FGH0 & UDP-glucose pyrophosphorylase \\
\hline W5FXY6 & $\begin{array}{l}\text { 26S proteasome non-atpase regulatory } \\
\text { subunit } 3\end{array}$ & D8L9K9 & Fructose-1,6-bisphosphatase, cytosolic,putative \\
\hline W5DW28 & $\begin{array}{l}\text { 26S proteasome non-atpase regulatory } \\
\text { subunit } 4\end{array}$ & W5HCE5 & Starch synthase I \\
\hline A0A077RXS4 & $\begin{array}{l}\text { 26S protease regulatory subunit } 6 \mathrm{~A} \\
\text { homolog }\end{array}$ & W5HA05 & Fructose-bisphosphate aldolase cytoplasmic isozyme \\
\hline P31251 & Ubiquitin-activating enzyme E1 2-like & C1J959 & Fructose-bisphosphate aldolase cytoplasmic isozyme \\
\hline W5B2T7 & Ubiquitin-activating enzyme E1 3 & W5HZ47 & Fructose-bisphosphate aldolase isozyme 1 \\
\hline W5DFQ0 & $\begin{array}{l}\text { Ubiquitin-like 1-activating enzyme } \\
\text { E1A }\end{array}$ & A0A096UTL2 & Glyceraldehyde-3-phosphate dehydrogenase \\
\hline W5FKD1 & $\begin{array}{l}\text { Ubiquitin-conjugating enzyme E2 } \\
\text { variant 1C-like }\end{array}$ & A0A0C4BJ74 & $\begin{array}{l}\text { NADP-dependent glyceraldehyde-3-phosphate } \\
\text { dehydrogenase }\end{array}$ \\
\hline W5CBQ9 & $\begin{array}{l}\text { Ubiquitin-conjugating enzyme E2-17 } \\
\text { kda }\end{array}$ & W5EAJ4 & Glucose phosphomutase \\
\hline W5D2H3 & Ubiquitin-conjugating enzyme E2 27 & W5B486 & Xylulose kinase \\
\hline W5BWF3 & E3 ubiquitin- ligase HERC4 & A0A077RSE3 & Pyruvate kinase, cytosolic isozyme \\
\hline W5AFH9 & $\begin{array}{l}\text { E3 ubiquitin- ligase MARCH11-like } \\
\text { isoform } \times 2\end{array}$ & W5FPI0 & Enolase 2-like isoform $\times 2$ \\
\hline W5H4P2 & DNA damage-inducible protein 1 & W5BG19 & 6-phosphogluconolactonase 2 \\
\hline W5EMB1 & Cell division cycle 48 & W5HDD8 & Sucrose synthase \\
\hline A0A068AZ53 & $\begin{array}{l}\text { WD-40 repeat-protein containing } \\
\text { MSI4-like }\end{array}$ & W5HXV0 & Fructokinase-2 \\
\hline $\begin{array}{l}\text { Ribosome } \\
\text { assembly/translation }\end{array}$ & & W5B4C2 & 4-alpha-glucanotransferase DPE2-like \\
\hline Q7XY23 & 40S ribosomal S3a-like & W5DRH6 & Cellulose synthase \\
\hline W5F4L4 & 40S ribosomal S29 & A0A096UMJ7 & Cellulose synthase- 4 \\
\hline A0A077S4G0 & 40S ribosomal S2 & Stress/defence & \\
\hline W5I1R7 & 40S ribosomal S3 & E9NVM6 & Group 3 late embryogenesis abundant \\
\hline U5HTD8 & 40 S ribosomal S20 & A0A096UP27 & Wheat cold induced 16 \\
\hline W5G3X9 & 40 S ribosomal S20 & W5DJR4 & Salt stress root RS1-like \\
\hline W5AUH7 & $40 \mathrm{~S}$ ribosomal $\mathrm{S} 10$ & A0A077RZB4 & Salt stress root RS1-like \\
\hline Q5I7L0 & 60 S ribosomal L18 & P46524 & Dehydrin WZY1-2 \\
\hline W5GGF8 & 60S ribosomal L13-2 & P93608 & Dehydrin WZY1-2 \\
\hline W5DEJ5 & 60S ribosomal L28-1-like & A7VL25 & Group3 late embryogenesis abundant \\
\hline W5CCH5 & 60S ribosomal L28-1-like & W5BA01 & Early responsive to dehydration \\
\hline W5F905 & 60 S ribosomal L7a & A0A077RRB6 & $\begin{array}{l}\text { Glutaredoxin domain-containing cysteine-rich } \\
\text { CG12206-like isoform } 1\end{array}$ \\
\hline W5FIP7 & 60S ribosomal L4-1-like & P93612 & Dehydrin 13 \\
\hline W5EW55 & 60S ribosomal L5-2 & W5HHN0 & Disease resistance RGA3 \\
\hline A0A077RTE5 & 60S ribosomal L9 & W5G7J3 & Temperature-induced lipocalin-1 \\
\hline W5C3Q8 & 60 S ribosomal L9 & W5FDB5 & Disease resistance protein RGA2 \\
\hline A0A0C4BJE3 & 60S ribosomal L10a-1 & A0A096ULF2 & Probable glutathione S-transferase GSTU6-like \\
\hline Q5I7L3 & 60S ribosomal L10a-1 & W5DYG7 & Superoxide dismutase [cu-zn] 2-like \\
\hline W5HQA2 & $60 S$ acidic ribosomal P1 & W5AJ14 & Thioredoxin $\mathrm{H}$ \\
\hline Q5I7L1 & $\begin{array}{l}\text { The large subunit ribosomal proteins } \\
\text { L13a }\end{array}$ & Q7FT21 & Thioredoxin $\mathrm{H}$ \\
\hline Q6EZA4 & Large subunit ribosomal protein $\mathrm{L} 40 \mathrm{e}$ & W5AMQ5 & Thioredoxin $\mathrm{H}$ \\
\hline W5A9E1 & Ubiquitin-40S ribosomal protein S27a & W5EDY2 & Glutathione peroxidase 4 \\
\hline $\begin{array}{l}\text { Chromatin-associated } \\
\text { processes }\end{array}$ & & W5ETS3 & Catalase 2 \\
\hline W5BYT5 & Histone H1 & Singal pathway & \\
\hline O65795 & Histone H1 & W5BMZ7 & Leucine-rich repeat transmembrane kinase 2 \\
\hline Q9XHL9 & Histone H1 & W5I1S6 & Calcium-binding CML8 \\
\hline W5FG65 & Histone H1 & A0A096US65 & Serine/threonine-protein kinase CTR1 \\
\hline O65794 & Histone $\mathrm{H} 1$ & W5GZ92 & $\begin{array}{l}\text { Seven transmembrane domain-containing tyrosine- } \\
\text { protein kinase } 1\end{array}$ \\
\hline W5A645 & Histone H2A & W5BCP7 & SAUR11 - auxin-responsive SAUR family member \\
\hline
\end{tabular}




\begin{tabular}{|c|c|c|c|}
\hline Accession no. ${ }^{a}$ & Proteins & Accession no. ${ }^{a}$ & Proteins \\
\hline W5DZG1 & Histone $\mathrm{H} 2 \mathrm{~A}$ & W5E096 & Rho guanine nucleotide exchange factor 6 \\
\hline W5HA06 & Histone H2B.3 & W5FB99 & Phosphatase $2 \mathrm{~A}$ isoform 2 (PP2Ac-2) \\
\hline W4ZWK0 & Histone $\mathrm{H} 2 \mathrm{~A}$ & K4MQ41 & Calcium-dependent kinase \\
\hline W5A444 & Histone H2B.2-like isoform 1 & A0A0C4BJH4 & 14-3-3 GF14-D-like \\
\hline A0A096UKH0 & Histone H2A.1 & L0GDQ5 & 14-3-3 B \\
\hline W5GES9 & Histone H2B.2 & L0GED8 & 14-3-3 expressed \\
\hline W5GGF1 & Histone H2B.2 & P04464 & Calmodulin \\
\hline W5H5H3 & Histone H2B.2 & Transport & \\
\hline A0A096UKE9 & H2B2_WHEAT ame: Full = histone & W5B354 & Protein transport protein sec. 24 -like \\
\hline A0A096UTK7 & Histone H2B.2 & W5DXD2 & Polyol transporter 4 \\
\hline W5HZ15 & Histone H2B.2 & A0A077RF08 & Bidirectional sugar transporter SWEET1a-like \\
\hline W5GH89 & Histone H2A & W5FZC0 & Sugar transport 14-like \\
\hline W5FGP3 & Histone $\mathrm{H} 2 \mathrm{~A}$ & A0A0E3IHU6 & Inorganic phosphate transporter 1-4 \\
\hline Q43312 & Histone H2A7 & W4ZRP5 & TOM1-like protein 2 \\
\hline W5BNS0 & Histone H2A.4 & W5HYJ9 & Nitrate/chlorate transporter \\
\hline A0A096USW9 & H2B2 WHEAT ame: Full = histone & W5HN28 & Lysine histidine transporter 1-like \\
\hline W5DZU8 & Histone -like isoform 1 & C7C5T9 & Sulfate transporter -like \\
\hline W5EA78 & Histone -like isoform 1 & W5H0U1 & TOM1-like protein 2 \\
\hline W5E0R4 & Histone -like isoform 1 & W5E117 & Low affinity sulfate transporter 3-like \\
\hline W4ZTZ4 & Histone -like isoform 1 & W5D0X5 & Amino acid permease \\
\hline A0A0C4BKM5 & Histone $\mathrm{H} 2 \mathrm{~A}$ variant 1 & Photosynthesis & \\
\hline W5F1A1 & Histone $\mathrm{H} 2 \mathrm{~A}$ variant 3 & P83970 & Plasma membrane $\mathrm{H}+$-atpase \\
\hline W5A7J0 & Histone $\mathrm{H} 3$ & Q2L9B8 & Vacuolar ATP synthase subunit E \\
\hline A0A096USG9 & Histone H4 & W5APB1 & Oxygen-evolving enhancer chloroplastic \\
\hline Protein folding & & A0A077RAG2 & Cytochrome b5 \\
\hline A7LM55 & Cyclophilin 1 & P69415 & Photosystem I subunit VII \\
\hline W5A8B5 & Heat shock $70 \mathrm{kda} 4 \mathrm{~L}$ & W5E7J2 & Psbp chloroplastic-like protein \\
\hline F4Y592 & Heat shock protein 90 & W5DWY0 & Psbp chloroplastic-like protein \\
\hline F4Y595 & Heat shock protein 90 & W5BG62 & $\begin{array}{l}\text { Ribulose 1,5-bisphosphate carboxylase/oxygenase } \\
\text { large subunit }\end{array}$ \\
\hline W5FEE5 & $\begin{array}{l}\text { Heat shock cognate } 70 \text { kda protein } \\
\text { 1-like isoform } 1\end{array}$ & W5HZ47 & Fructose-bisphosphate aldolase isozyme 1 \\
\hline W5BDM2 & Heat shock cognate 70 kda protein 1 & C1J959 & Fructose-bisphosphate aldolase cytoplasmic isozyme \\
\hline W5DZG0 & Heat shock cognate 70 kda protein & W5HA05 & Fructose-bisphosphate aldolase cytoplasmic isozyme \\
\hline Q9SAU8 & HSP70 & D8L9K9 & Fructose-1,6-bisphosphatase, cytosolic,putative \\
\hline F8RP11 & HOP & A0A096UTL2 & Glyceraldehyde-3-phosphate dehydrogenase \\
\hline Cytoskeleton building & & $\begin{array}{l}\text { Mitochondrial } \\
\text { fission }\end{array}$ & \\
\hline W5EGW3 & Kinesin-4 & W4ZR59 & Mitochondria fission 1 protein \\
\hline W5DRH6 & Cellulose synthase & \begin{tabular}{|l|l|}
$\begin{array}{l}\text { Cell cycle } \\
\text { progression }\end{array}$ \\
\end{tabular} & \\
\hline A0A096UMJ7 & Cellulose synthase- 4 & A0MA43 & Ran-binding protein \\
\hline W5ETI1 & Actin-1 & $\begin{array}{l}\text { mRNA } \\
\text { Processing }\end{array}$ & \\
\hline A0A0A7NVN8 & Actin depolymerizing factor & W5ADX0 & Pre-mRNA-processing factor 19-2-like protein \\
\hline W5BLI5 & Mixed-linked glucan synthase 3 & \begin{tabular}{|l|l|}
$\begin{array}{l}\text { Sulfur } \\
\text { metabolism }\end{array}$ \\
\end{tabular} & \\
\hline Fatty acid metabolism & & W5FLB0 & Cysteine synthase \\
\hline A0A077RUB2 & Phospholipase A1-II 5-like & Apoptosis & \\
\hline W5DS68 & Lipoxygenase 3 & W5GE21 & Apoptosis-inducing factor-like protein A \\
\hline W5FLT9 & Long-chain-fatty-acid-coa ligase 4 & $\begin{array}{l}\text { Nitrogen } \\
\text { metabolism }\end{array}$ & \\
\hline A0A096UUN4 & Delta-12 oleate desaturase & Q45NB6 & Glutamine synthetase isoform GS1b \\
\hline W5E0C4 & 3-ketoacyl-coa synthase 6 & RNA edit & \\
\hline $\begin{array}{l}\text { Membrane-associated } \\
\text { proteins }\end{array}$ & & W5CBR8 & $\begin{array}{l}\text { Pentatricopeptide repeat-containing mitochondrial- } \\
\text { like (PPR) }\end{array}$ \\
\hline A0A077S2A9 & Patellin-5-like & \begin{tabular}{|l|}
$\begin{array}{l}\text { Flavonoid } \\
\text { metabolism }\end{array}$ \\
\end{tabular} & \\
\hline W5ASB4 & Aquaporin 8 & W5BZL1 & Chalcone synthase \\
\hline
\end{tabular}




\begin{tabular}{|l|l|l|l|}
\hline Accession no. $^{\text {a }}$ & Proteins & Accession no. $^{\mathbf{a}}$ & Proteins $^{-1}$ \\
\hline A7J2I2 & Plasma membrane intrinsic protein & DNA repair & \\
\hline A7J2I3 & Plasma membrane intrinsic protein & W5FH53 & DNA repair RAD23 \\
\hline Q9M7C4 & Plasma membrane intrinsic protein 1 & W5FZL2 & DNA repair RAD23 \\
\hline W5FVE5 & Probable aquaporin PIP2-7-like & W5HG98 & DNA repair RAD23 \\
\hline
\end{tabular}

Table 1. Examples of pathways with ubiquitylation targets. ${ }^{a}$ Accession no.: accession number in Uniprot database. See Additional file 4 for the complete list (according to the GO annotation).
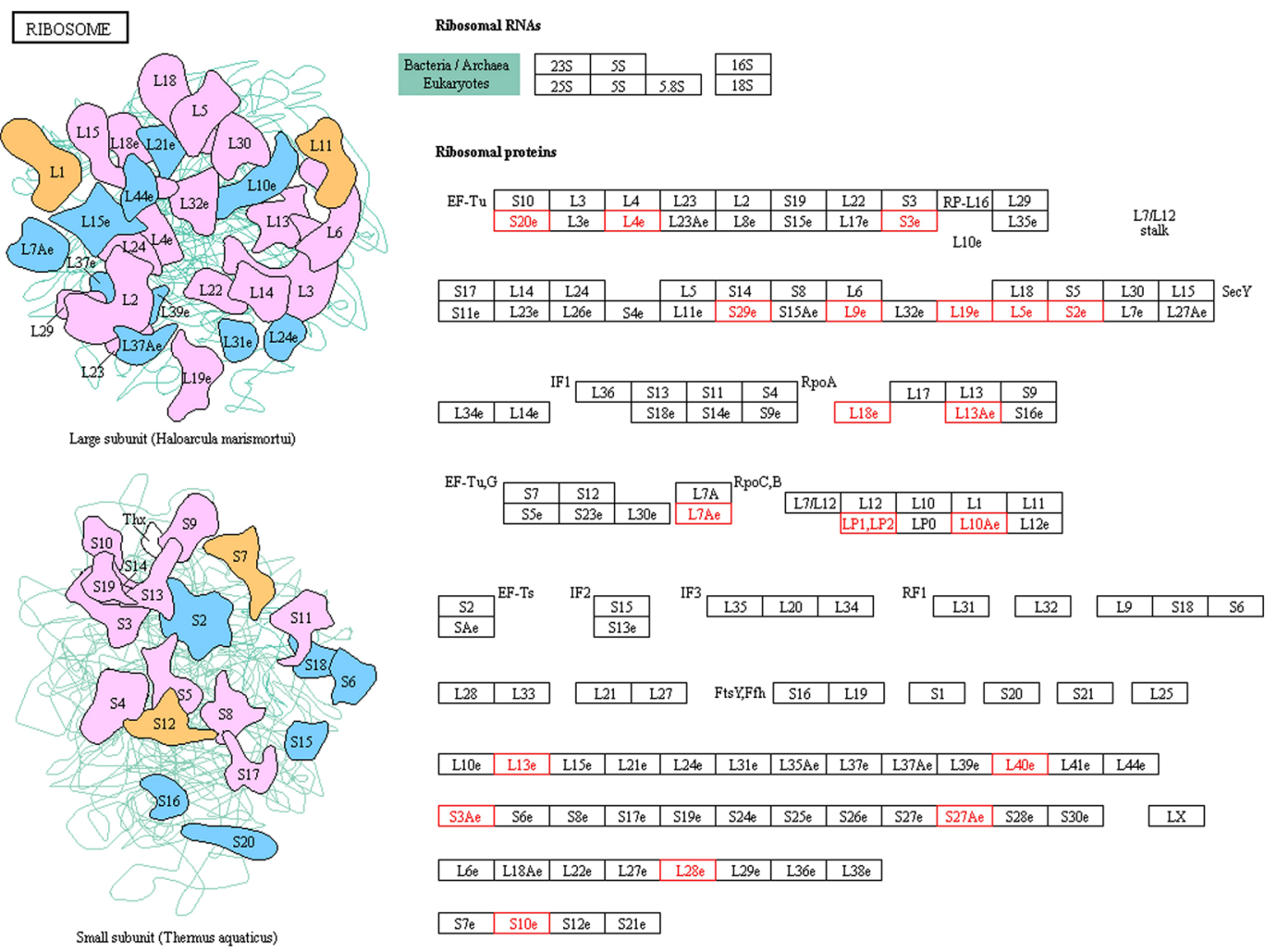

\begin{tabular}{|l|l|l|l|l|l|l|l|l|l|l|l|l|}
\hline L10e & L13e & L15e & L21e & L24e & L31e & L35Ae & L37e & L37Ae & L39e & L40e & L41e & L44e \\
\hline
\end{tabular}

\begin{tabular}{|l|l|l|l|l|l|l|l|l|l|l|l|}
\hline S3Ae & S6e & S8e & S17e & S19e & S24e & S25e & S26e & S27e & S27Ae & S28e & S30e \\
\hline
\end{tabular}

\begin{tabular}{|l|l|l|l|l|l|l|l|}
\hline L6e & L18Ae & L22e & L27e & L28e & L29e & L36e & L38e \\
\hline
\end{tabular}

\begin{tabular}{|l|l|l|l|}
\hline S7e & S10e & S12e & S21e \\
\hline
\end{tabular}

Figure 6. Ubiquitinated subunits of the $40 \mathrm{~S}$ and $60 \mathrm{~S}$ ribosome complexes in wheat. Ubiquitinated subunits are highlighted in red. Source: Kanehisa et al. ${ }^{26}$.

showed that a total of 220 ubiquitinated proteins were mapped to the protein interaction database, which presents how ubiquitylated proteins perform diverse functions in wheat. This network identified a complex interconnected web with a number of ubiquitylated proteins present at key hubs, with subnetworks involved in carbohydrate metabolism, sugar synthesis, ribosome assembly/translation, $26 \mathrm{~S}$ proteasome composition, membrane transport (aquaporins and $\mathrm{H}+$-ATPases), and chromatin-associated processes being especially enriched (Fig. 8). For example, among the clusters in the network, Cluster I-II consist of proteins involved in ribosome-associated and carbohydrate metabolism, while Cluster III refers to various ubiquitylated histones that may play important roles in regulation of chromatin-associated processes. The clusters of these three pathways all displayed dense protein interaction networks. The physiological interactions among these proteins may lead to their cooperation and coordination in wheat.

Conservation analysis of the ubiquitinated proteins. To reveal the commonality and specificity of lysine ubiquitination between wheat and other species, we used the sequences of the identified proteins to perform a BLAST search and estimated the degree of conservation of ubiquitinated proteins among wheat, rice, and Arabidopsis thaliana. The parameters were set as follows: E-value $<1 \times 10^{-10}$, score $\geq 80$, and identity $\geq 30 \%$. As shown in Fig. 9A, 206 (72.3\%) of the identified ubiquitinated proteins in wheat had orthologous proteins in the other two species. A total of 83 ubiquitinated proteins were found in all three species (Additional file 7; Fig. 9A). Further analysis demonstrated that most of these orthologous proteins were involved in signal pathway, carbon metabolism, chromatin-associated processes, $26 \mathrm{~S}$ proteasome, and protein folding (Additional file 8; Fig. 9B). Among the 285 identified ubiquitinated proteins in wheat, 31 had conserved orthologs with an average identity of $80.9 \%$ in Arabidopsis thaliana (Additional file 7; Fig. 9A), which involved in ribosomal proteins, 26S proteasome subunits and cytoskeleton building proteins. However, 92 had highly conserved orthologs with an average identity of $84.6 \%$ in rice (Additional file 7; Fig. 9A), and most of these proteins participated in chromatin-associated processes and stress/ 


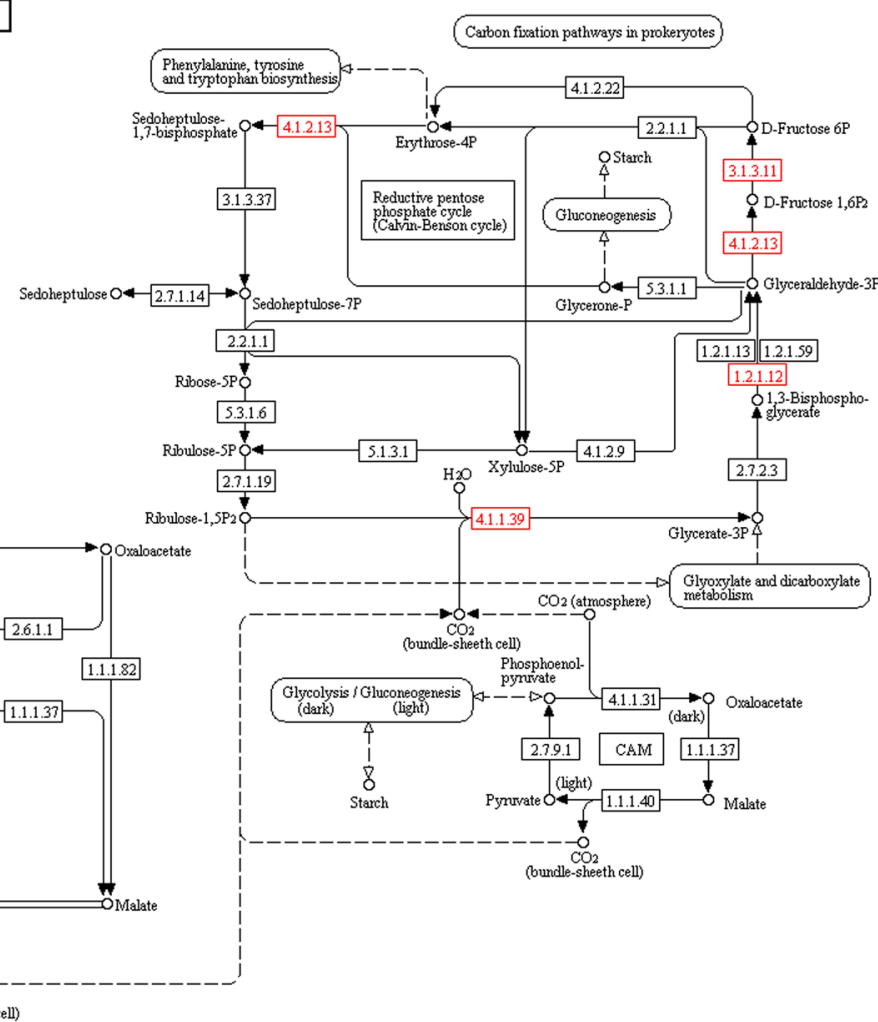

Figure 7. Ubiquitinated proteins in representative metabolic pathways in terms of carbon fixation in photosynthetic organisms in wheat. Ubiquitinated proteins are highlighted in red. W5HZ47, C1J959 and W5HA05 corresponds to 4.1.2.13, W5BG62 corresponds to 4.1.1.39, D8L9K9 corresponds to 3.1.3.11, and A0A096UTL2 corresponds to 1.2.1.12. Source: Kanehisa et al. ${ }^{26}$.

defence. This indicated that ubiquitination is conserved in different species and plays important roles in signal pathway and chromatin-associated processes. In addition, 79, 229, and 765 ubiquitinated proteins were only found in wheat, rice, and Arabidopsis thaliana, respectively (Additional file 7). For instance, ubiquitinated proteins associated with mitochondrial fission, apoptosis and cell cycle progression were only identified in wheat (Table 1). These results imply that lysine ubiquitination plays both common and specific roles in different plant species.

\section{Discussion}

Strategy for ubiquitome in wheat. Protein conjugation with ubiquitin, known as ubiquitination, is a well conserved post-translational modification in eukaryotic organisms that plays important roles in many cellular processes. Until recently, the ubiquitome was only reported in two plant species, Arabidopsis and rice (Oryza sativa $)^{1,16-21}$. Obviously, the proteome-wide ubiquitination data are lacking for many other plant species. In this study, we conducted a proteomics study of lysine ubiquitination in common wheat, which is one of the most important crops in the world. Through combining the highly specific enrichment of lysine-ubiquitinated peptides with highly sensitive LC-MS/MS, 433 lysine modification sites were identified on 285 proteins.

Previous studies mainly focused on either the use of overexpression of epitope-tagged ubiquitin or ubiquitin binding domains/antibodies to capture ubiquitylated proteins for identification by MS ${ }^{11-13,33,34}$. However, the low occupancy of ubiquitylation challenges detection of endogenously modified proteins in the absence of overexpression of either ubiquitin or substrate ${ }^{15}$. A highly robust and streamlined proteomic method to map endogenous putative ubiquitylation sites precisely was utilized in this study. The advantages over previously described MS-based methods for ubiquitylation sites identification are as follows: (1) it can identify ubiquitylation sites in any tissue or organism; (2) it analyzes endogenous ubiquitylation sites in-depth on the proteome-wide level; (3) it is highly efficient at enriching the ubiquitylated peptides, enabling detection of low abundant modification sites; (4) it only requires a single-step affinity enrichment of modified peptides; (5) it is fully compatible with proteome-wide site-specific quantification of ubiquitylation.

Ubiquitylation regulates diverse biological processes. The ubiquitinated proteins identified in this study belong to diverse functional groups based on their GO annotations and are localized to multiple cellular compartments, indicating that lysine ubiquitination plays important roles in regulating many cellular processes in wheat. Protein interaction network analysis demonstrated that a broad range of interactions are modulated by protein ubiquitination. Furthermore, the conservation analysis of the ubiquitinated proteins between wheat and 


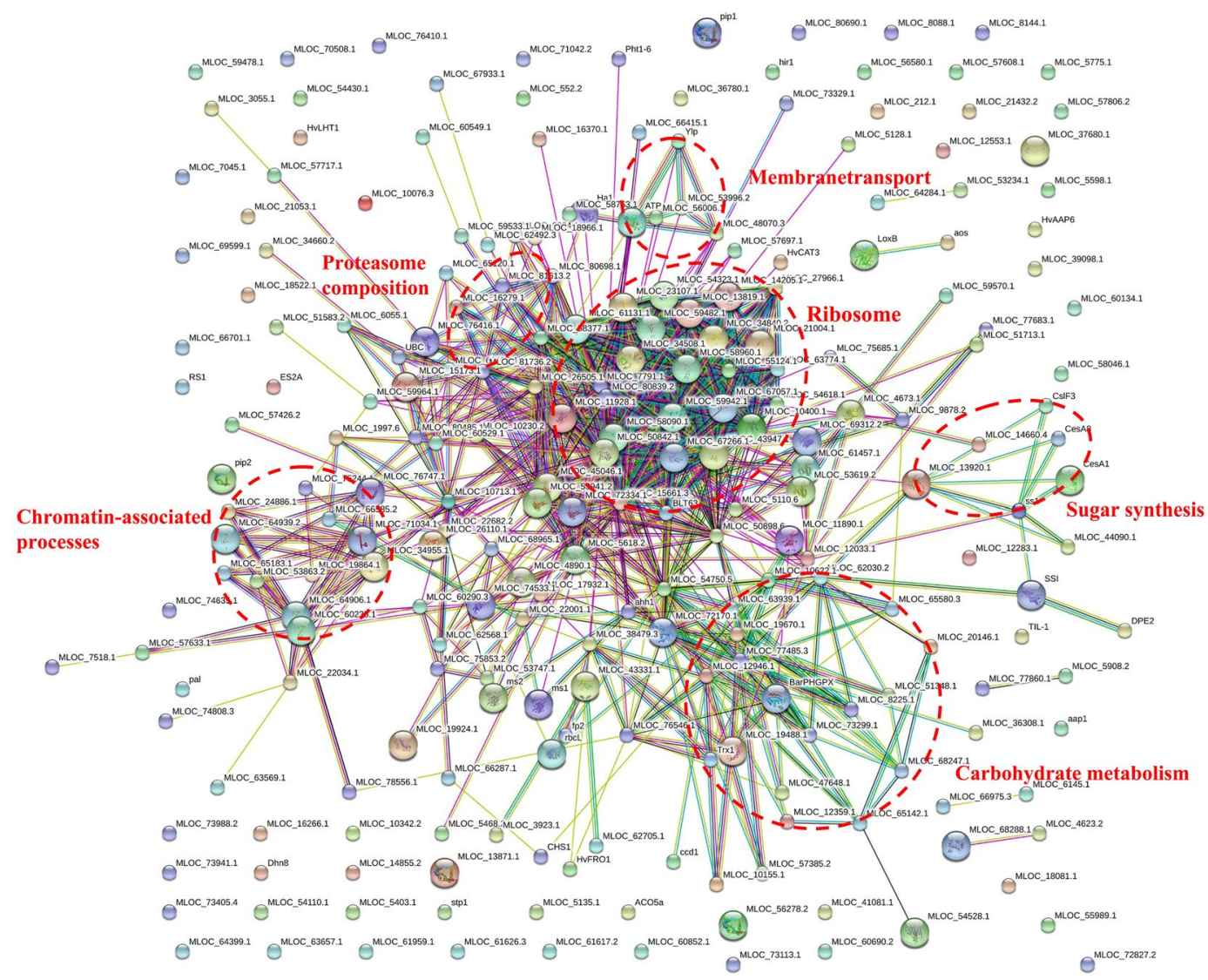

Figure 8. Interaction networks of the ubiquitinated proteins in wheat using String software. Different colored lines represent types of evidence for association: green, neighborhood evidence; red, fusion evidence; purple, experimental evidence; light blue, database evidence; black, coexpression evidence; blue, co-occurrence evidence; and yellow, text-mining evidence.

A

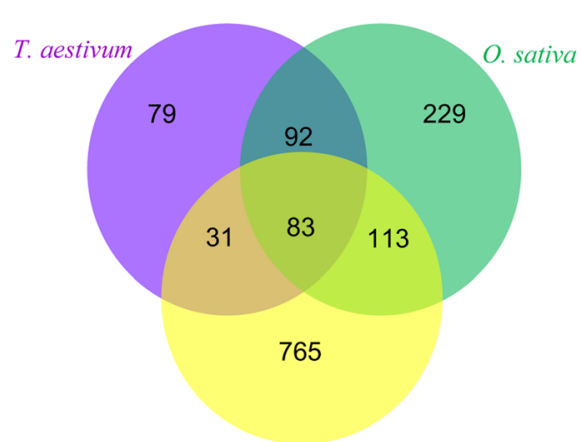

A. thaliana

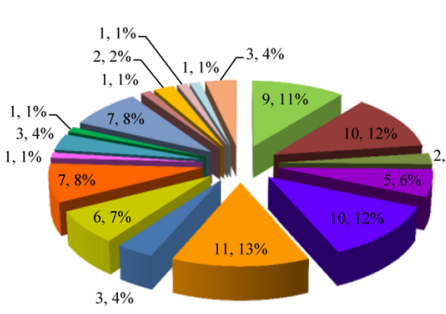

- Carbohydrate metabolism

- Ribosome assembly/translation

- Stress/defence

- Chromatin-associated processes

= Singal pathway

- Transport

= Photosynthesis

- Protein folding

= Phenylpropanoid metabolism

$=$ Protein synthesis

- Fatty acid metabolism

- Membrane-associated proteins

- Sulfur metabolism

$=$ Cysteine and methionine metabolism

=Cyanoamino acid metabolism

= Retinol metabolism

- Other metabolisms

Figure 9. Conservation analysis of the ubiquitinated proteins in wheat. (A) All the identified ubiquitinated proteins in wheat compared with Oryza sativa, Arabidopsis thaliana. (B) Functional classification of the common ubiquitinated proteins identified in wheat, Oryza sativa, and Arabidopsis thaliana.

other species imply that lysine ubiquitination displays both commonality and specificity in different plant species. This study provides the first comprehensive view of the ubiquitome in wheat.

In the $26 \mathrm{~S}$ proteasome, four core subunits (W5BUE8, W5FXY6, W5DW28, A0A077RXS4) were found to be ubiquitylated. Additionally, other UPS components including the ubiquitin-like 1-activating enzyme E1A (W5DFQ0), ubiquitin-conjugating enzyme E2 27 (W5D2H3), E3 ubiquitin-ligase MARCH11-like isoform X2 (W5AFH9), DNA damage-inducible protein 1 (Ddi1) (W5H4P2), cell division cycle 48 (CDC48) (W5EMB1), and WD-40 repeat-protein O (A0A068AZ53) were notably enriched in our wheat ubiquitylome catalog, similar 
to the results of studies investigating other eukaryotes ${ }^{5,7,12,15}$. One example is CDC48, which plays a central role in the UPS-dependent turnover of misfolded ER-resident proteins after their retrograde transport back to the cytosol $^{35}$ as reported to be ubiquitylated in yeast ${ }^{11,36}$. Ubiquitinated proteins are either directly recognized by the proteasomal Ub receptors or first bound by the so-called shuttle proteins, which then drive the ubiquitinated proteins to the $26 \mathrm{~S}$ proteasome for degradation ${ }^{37-39}$. Ddil belongs to a family of shuttle proteins targeting polyubiquitinated substrates for proteasomal degradation ${ }^{40}$. One Ddil identified in this study was also found in rice ubiquitome ${ }^{16}$. However, E3 ubiquitin-ligase MARCH11-like isoform $\times 2$ and WD- 40 repeat-protein $\mathrm{O}$ were first identified in wheat. These findings suggest that such autoubiquitylation has regulatory consequences or might simply reflect collateral damage caused by their proximity to the Ub-transfer machinery.

Evidence of ribosomal subunits being ubiquitylated has previously been presented for Arabidopsis ${ }^{17,20}$ and other eukaryotes $5,7,11,15,41$. This modification could represent a regulatory step during ribosome assembly and/or translation, or implicate the UPS in the removal of improperly folded subunits or entire ribosomes when they become non-functional or less important upon cell starvation ${ }^{42}$. In this study, a large set of ubiquitylated subunits of the $40 \mathrm{~S}$ and $60 \mathrm{~S}$ ribosome complexes in wheat were identified (Fig. 6), implying that ubiquitination is likely to be an important regulatory mechanism for ribosomal proteins. Ubiquitylation plays important roles in protein trafficking and membrane protein turnover ${ }^{43,44}$. In this study, the presence of $\mathrm{H}+$-ATPase, aquaporin, intrinsic protein, and patellin in the ubiquitylome catalog suggest a vital role for $\mathrm{Ub}$ in controlling plasma membrane protein activity and/or turnover in wheat, likely via an endocytosis pathway that extracts the receptors from the membrane and delivers them to the vacuole for turnover ${ }^{17}$. A previous study indicated the expression of a fusion between $\mathrm{H}+$-ATPase AHA1 and Ub in Arabidopsis was sufficient to induce its endocytosis and sorting into the vacuolar lumen ${ }^{45}$. Furthermore, a previous study in other eukaryotes indicated that histones or isoforms were modified by PTMs, such as phosphorylation, acetylation and methylation, and ubiquitination, which are known to play important roles in the regulation of chromatin-associated processes ${ }^{46}$. We identified some ubiquitylation sites on various histones, including five major histones ( $\mathrm{H} 1, \mathrm{H} 2 \mathrm{~A}, \mathrm{H} 2 \mathrm{~B}, \mathrm{H} 3$, and $\mathrm{H} 4)$, several isoforms of histone, and two histone $\mathrm{H} 2 \mathrm{~A}$ variants. Generally, these results demonstrate the importance of ubiquitylation and the UPS in the catabolism and trafficking of membrane-associated proteins.

Carbohydrate metabolism regulates sugar synthesis and transformation as well as carbon partitioning. The process is a highly critical metabolic pathway in plants ${ }^{47}$. In this study, a large number of ubiquitylation sites were observed on carbohydrate metabolism-related enzymes. Notable examples in carbohydrate metabolism include those that play important roles in glycolysis, such as glyceraldehyde-3-phosphate dehydrogenase (A0A096UTL2, A0A0C4BJ74), enolase 2-like isoform $\times 2$ (W5FPI0), the cytosolic isozymes of pyruvate kinase (A0A077RSE3) and fructose-bisphosphate aldolase (C1J959, W5HA05, W5HZ47); and those that occupy key cytosolic steps in sugar formation and metabolism, such as starch synthase I (W5HCE5), glucose phosphomutase (W5EAJ4), sucrose synthase (W5HDD8), fructokinase-2 (W5HXV0), 4-alpha-glucanotransferase DPE2-like (W5B4C2) and UDP-glucose pyrophosphorylase (W5FGH0); as well as those that is vital in pentose phosphate pathway (PPP), such as xylulose kinase (W5B486), and 6-phosphogluconolactonase 2 (W5BG1). In support of our findings, the majority of the enzymes found to be related carbohydrate metabolism in this study were lysine ubiquitylated in Arabidopsis and rice ${ }^{48,49}$. These findings highlight the notion that lysine ubiquitylation plays a key regulatory role in the process of carbohydrate metabolism.

Diverse cell surface receptors and intermediate signaling components in this study were found to be ubiquitylated. In plants, receptor tyrosine kinases (RLKs) mediate many signaling messages at the cell surface and act as key regulators during developmental processe ${ }^{50}$. A previous study indicated that ubiquitylation of RTKs controls the amplitude and duration of receptor signaling ${ }^{51}$. We also mapped several ubiquitylation sites on tyrosine-protein kinase (RTKs) (W5GZ92) and leucine-rich repeat transmembrane kinase 2 (LRR-RLKs) (W5BMZ7). Other cell surface receptors and intermediate signaling components, such as 14-3-3, which function as homodimers or heterodimers and bind a large number of differentially phosphorylated substrates to regulate a wide array of cellular signaling and physiological processes, were ubiquitylated. These include calmodulin, an intracellular target of the secondary messenger $\mathrm{Ca}^{2+}$ that acts as part of a calcium signal transduction pathway by modifying its interactions with various target proteins such as kinases or phosphatases ${ }^{52,53}$. The serine/ threonine-protein kinase CTR1, Rho guanine nucleotide exchange factor 6, phosphatase 2A isoform 2 (PP2Ac2), calcium-dependent kinase (CDPKs), and auxin-responsive SAUR family members were also ubiquitylated. These proteins are involved in a variety of signaling pathways such as the calcium signaling pathway, PI3K-Akt signaling pathway, ethylene signal transduction, and abscisic acid, auxin hormone signaling. The majority of cell surface receptors and intermediate signaling components are also present in Arabidopsis thaliana ${ }^{17,54}$ and human ubiquitome ${ }^{15}$; however, auxin-responsive SAUR family member and Rho guanine nucleotide exchange factor 6 have not been reported previously. The identification of ubiquitylation sites on cell surface receptors and major components of the signal pathways indicates that ubiquitylation plays a broad regulatory role in these signaling pathways in wheat. These findings also suggest that Ub addition controls the crosstalk between pathways.

Proteins involved in photosynthesis were identified in the wheat ubiquitome. The conversion of light energy to chemical energy in photosynthesis involves electron transfer and ATP synthesis. Driven by light, photosystem II (PSII) catalyzes electron transfer from water to plastoquinone. PsbP is a thylakoid luminal subunit of photosystem II (PSII) that plays an important role in maintaining photosynthetic electron transfer ${ }^{54}$. Unlike PsbP proteins, PsbP-like protein has been implicated in the assembly, stability, and/or repair of PSII complexes or subunits ${ }^{55}$. We mapped ubiquitylation sites on 2 psbP-like proteins (W5E7J2 and W5DWY0). Additionally, other components important for electron transfer such as cytochrome b5 (Cyt b5) (A0A077RAG2), oxygen-evolving enhancer chloroplastic (W5APB1), and vacuolar ATP synthase subunit E (P83970) were identified as ubiquitylated proteins in wheat. These findings demonstrate that lysine ubiquitylation plays a key regulatory role in the process of photosynthesis.

Wheat provides a large amount of starch ever year that is synthesized from one product of photosynthesis, glu$\operatorname{cose}^{56}$. During photosynthesis, the Calvin cycle converts carbon dioxide and other compounds into glucose. Six 
metabolic enzymes in the Calvin cycle were found to be ubiquitinated in wheat (Fig. 7). Similar results were also found in the rice ubiquitome ${ }^{16}$. Rubisco is a major protein in the stroma of chloroplasts that fixes carbon dioxide as the first step of the Calvin cycle via catalyzing the carboxylation of ribulose-1,5-bisphosphate ${ }^{57}$. These studies suggest that, in plants, lysine ubiquitylation participates in regulation of the activity of enzymes in the Calvin cycle.

Ubiquitylated proteins were found to be participating in nuclear as well as nonnuclear processes, including DNA repair, apoptosis, and protein folding. For example, three DNA repair RAD23 (W5FH53, W5FZL2, and W5HG98) were modified by ubiquitylation. RAD23 is an evolutionarily conserved protein that is important for nucleotide excision repair ${ }^{58}$. The stabilization of DNA repair and stress factors could represent an important biological function of RAD23 $3^{59}$. A previous study also indicated that RAD23 links DNA repair to the ubiquitin/ proteasome pathway ${ }^{58}$. Moreover, one apoptosis-inducing factor-like protein A (W5GE21) and two heat shock proteins (HSP 90 and HSP70) were found to be ubiquitylated. Particularly notable, we found numerous ubiquitylated proteins involved in stress/defense, transport, nitrate metabolism, sulfur metabolism, fatty acid metabolism, cell wall metabolism, flavonoid metabolism, mitochondrial fission, cell cycle progression, mRNA processing (Table 1) and other metabolic processes, suggesting that these processes are heavily influenced by Ub addition. Besides, some ubiquitylated proteins were identified for the first time in this study.

Overall, this study provides the first extensive data on lysine ubiquitination in common wheat, and enhances the concept that ubiquitination mediates diverse cellular processes, especially with respect to proteasome composition, ribosome assembly/translation, carbohydrate metabolism, signal transduction, and photosynthesis. Our findings broaden the extent of physiological processes regulated by lysine ubiquitination and will serve as a valuable reference for the functional analysis of lysine ubiquitination in wheat and other plants.

\section{References}

1. Kim, D. Y., Scalf, M., Smith, L. M. \& Vierstra, R. D. Advanced proteomic analyses yield a deep catalog of ubiquitylation targets in Arabidopsis. Plant Cell 25, 1523-40 (2013).

2. Kwon, S. J., Choi, E. Y., Choi, Y. J., Ahn, J. H. \& Park, O. K. Proteomics studies of post-translational modifications in plants. J Exp Bot 57, 1547-51 (2006)

3. Dikic, I., Wakatsuki, S. \& Walters, K. J. Ubiquitin-binding domains - from structures to functions. Nat Rev Mol Cell Biol 10, 659-71 (2009).

4. Jiang, X. \& Chen, Z. J. The role of ubiquitylation in immune defence and pathogen evasion. Nat Rev Immunol 12, 35-48 (2011).

5. Kim, W. et al. Systematic and quantitative assessment of the ubiquitin-modified proteome. Mol Cell 44, 325-40 (2011).

6. Sadanandom, A., Bailey, M., Ewan, R., Lee, J. \& Nelis, S. The ubiquitin-proteasome system: central modifier of plant signalling. New Phytol 196, 13-28 (2012).

7. Xu, G., Paige, J. S. \& Jaffrey, S. R. Global analysis of lysine ubiquitination by ubiquitin remnant immunoaffinity profiling. Nat Biotechnol 28, 868-73 (2010).

8. Dreher, K. \& Callis, J. Ubiquitin, hormones and biotic stress in plants. Ann Bot 99, 787-822 (2007).

9. Li, W. et al. The U-Box/ARM E3 ligase PUB13 regulates cell death, defense, and flowering time in Arabidopsis. Plant Physiol 159, 239-50 (2012).

10. Yates, G. \& Sadanandom, A. Ubiquitination in plant nutrient utilization. Front Plant Sci 4, 452 (2013).

11. Peng, J. et al. A proteomics approach to understanding protein ubiquitination. Nat Biotechnol 21, 921-6 (2003).

12. Danielsen, J. M. et al. Mass spectrometric analysis of lysine ubiquitylation reveals promiscuity at site level. Mol Cell Proteomics $\mathbf{1 0}$, M110 003590 (2011).

13. Meierhofer, D., Wang, X., Huang, L. \& Kaiser, P. Quantitative analysis of global ubiquitination in HeLa cells by mass spectrometry. $J$ Proteome Res 7, 4566-76 (2008).

14. Shi, Y. et al. A data set of human endogenous protein ubiquitination sites. Mol Cell Proteomics 10, M110 002089 (2011).

15. Wagner, S. A. et al. A proteome-wide, quantitative survey of in vivo ubiquitylation sites reveals widespread regulatory roles. Mol Cell Proteomics 10, M111 013284 (2011).

16. Xie, X., Kang, H., Liu, W. \& Wang, G. L. Comprehensive profiling of the rice ubiquitome reveals the significance of lysine ubiquitination in young leaves. J Proteome Res 14, 2017-25 (2015).

17. Saracco, S. A. et al. Tandem affinity purification and mass spectrometric analysis of ubiquitylated proteins in Arabidopsis. Plant J 59, 344-58 (2009).

18. Igawa, T. et al. Isolation and identification of ubiquitin-related proteins from Arabidopsis seedlings. J Exp Bot 60, 3067-73 (2009).

19. Manzano, C., Abraham, Z., Lopez-Torrejon, G. \& Del Pozo, J. C. Identification of ubiquitinated proteins in Arabidopsis. Plant Mol Biol 68, 145-58 (2008).

20. Maor, R. et al. Multidimensional protein identification technology (MudPIT) analysis of ubiquitinated proteins in plants. Mol Cell Proteomics 6, 601-10 (2007).

21. Liu, C. W. et al. Proteomic analysis of salt-responsive ubiquitin-related proteins in rice roots. Rapid Commun Mass Spectrom 26, 1649-60 (2012).

22. Zhang, N., Chen, F., Huo, W. \& Cui, D. Proteomic analysis of middle and late stages of bread wheat (Triticum aestivum L.) grain development. Front Plant Sci 6, 735 (2015).

23. Zhang, N., Huo, W., Zhang, L., Chen, F. \& Cui, D. Identification of Winter-Responsive Proteins in Bread Wheat Using Proteomics Analysis and Virus-Induced Gene Silencing (VIGS). Mol Cell Proteomics 15, 2954-69 (2016).

24. Vizcaino, J. A. et al. 2016 update of the PRIDE database and its related tools. Nucleic Acids Res 44, D447-56 (2016).

25. Weinert, B. T. et al. Proteome-wide mapping of the Drosophila acetylome demonstrates a high degree of conservation of lysine acetylation. Sci Signal 4, ra48 (2011).

26. Kanehisa, M., Furumichi, M., Tanabe, M., Sato, Y. \& Morishima, K. KEGG: new perspectives on genomes, pathways, diseases and drugs. Nucleic Acids Res 45, D353-D361 (2017).

27. Horton, P. et al. WoLF PSORT: protein localization predictor. Nucleic Acids Res 35, W585-7 (2007).

28. Tay, A. P., Pang, C. N. I., Winter, D. L. \& Wilkins, M. R. PTMOracle: A Cytoscape App for Covisualizing and Coanalyzing PostTranslational Modifications in Protein Interaction Networks. J Proteome Res 16, 1988-2003 (2017).

29. Zhen, S. et al. First Comprehensive Proteome Analyses of Lysine Acetylation and Succinylation in Seedling Leaves of Brachypodium distachyon L. Sci Rep 6, 31576 (2016).

30. Mergner, J. \& Schwechheimer, C. The NEDD8 modification pathway in plants. Front Plant Sci 5, 103 (2014).

31. Zhang, D. \& Zhang, D. E. Interferon-stimulated gene 15 and the protein ISGylation system. J Interferon Cytokine Res 31, 119-30 (2011).

32. Kerscher, O., Felberbaum, R. \& Hochstrasser, M. Modification of proteins by ubiquitin and ubiquitin-like proteins. Annu Rev Cell Dev Biol 22, 159-80 (2006).

33. Matsumoto, M. et al. Large-scale analysis of the human ubiquitin-related proteome. Proteomics 5, 4145-51 (2005). 
34. Tagwerker, C. et al. A tandem affinity tag for two-step purification under fully denaturing conditions: application in ubiquitin profiling and protein complex identification combined with in vivocross-linking. Mol Cell Proteomics 5, 737-48 (2006).

35. Bar-Nun, S. The role of $\mathrm{p} 97 / \mathrm{Cdc} 48 \mathrm{p}$ in endoplasmic reticulum-associated degradation: from the immune system to yeast. Curr Top Microbiol Immunol 300, 95-125 (2005).

36. Hitchcock, A. L., Auld, K., Gygi, S. P. \& Silver, P. A. A subset of membrane-associated proteins is ubiquitinated in response to mutations in the endoplasmic reticulum degradation machinery. Proc Natl Acad Sci USA 100, 12735-40 (2003).

37. Kaplun, L. et al. The DNA damage-inducible UbL-UbA protein Ddil participates in Mec1-mediated degradation of Ho endonuclease. Mol Cell Biol 25, 5355-62 (2005).

38. Husnjak, K. et al. Proteasome subunit Rpn13 is a novel ubiquitin receptor. Nature 453, 481-8 (2008)

39. Hicke, L., Schubert, H. L. \& Hill, C. P. Ubiquitin-binding domains. Nat Rev Mol Cell Biol 6, 610-21 (2005).

40. Nowicka, U. et al. DNA-damage-inducible 1 protein (Ddil) contains an uncharacteristic ubiquitin-like domain that binds ubiquitin. Structure 23, 542-57 (2015).

41. Emanuele, M. J. et al. Global identification of modular cullin-RING ligase substrates. Cell 147, 459-74 (2011).

42. Kraft, C., Deplazes, A., Sohrmann, M. \& Peter, M. Mature ribosomes are selectively degraded upon starvation by an autophagy pathway requiring the Ubp3p/Bre5p ubiquitin protease. Nat Cell Biol 10, 602-10 (2008).

43. Mukhopadhyay, D. \& Riezman, H. Proteasome-independent functions of ubiquitin in endocytosis and signaling. Science 315, 201-5 (2007).

44. MacGurn, J. A., Hsu, P. C. \& Emr, S. D. Ubiquitin and membrane protein turnover: from cradle to grave. Annu Rev Biochem 81, 231-59 (2012)

45. Herberth, S. et al. Artificial ubiquitylation is sufficient for sorting of a plasma membrane ATPase to the vacuolar lumen of Arabidopsis cells. Planta 236, 63-77 (2012).

46. Suganuma, T. \& Workman, J. L. Crosstalk among Histone Modifications. Cell 135, 604-7 (2008).

47. Li, G. et al. Proteomic analysis of leaves and roots of common wheat (Triticum aestivum L.) under copper-stress conditions. J Proteome Res 12, 4846-61 (2013).

48. Kim, D.-Y., Scalf, M., Smith, L. M. \& Vierstra, R. D. Advanced Proteomic Analyses Yield a Deep Catalog of Ubiquitylation Targets in Arabidopsis. Plant Cell 25, 1523-1540 (2013).

49. Xie, X., Kang, H., Liu, W. \& Wang, G.-L. Comprehensive Profiling of the Rice Ubiquitome Reveals the Significance of Lysine Ubiquitination in Young Leaves. J Proteome Res 14, 2017-2025 (2015).

50. Johnson, K. L. \& Ingram, G. C. Sending the right signals: regulating receptor kinase activity. Curr Opin Plant Biol 8, 648-56 (2005).

51. Marmor, M. D. \& Yarden, Y. Role of protein ubiquitylation in regulating endocytosis of receptor tyrosine kinases. Oncogene 23, 2057-70 (2004).

52. Stevens, F. C. Calmodulin: an introduction. Can J Biochem Cell Biol 61, 906-10 (1983).

53. Chin, D. \& Means, A. R. Calmodulin: a prototypical calcium sensor. Trends Cell Biol 10, 322-8 (2000).

54. Ifuku, K., Ishihara, S., Shimamoto, R., Ido, K. \& Sato, F. Structure, function, and evolution of the PsbP protein family in higher plants. Photosynth Res 98, 427-37 (2008).

55. Lu, Y. Identification and Roles of Photosystem II Assembly, Stability, and Repair Factors in Arabidopsis. Front Plant Sci 7, 168 (2016).

56. Keeling, P. L., Wood, J. R., Tyson, R. H. \& Bridges, I. G. Starch Biosynthesis in Developing Wheat Grain:Evidence against the Direct Involvement of Triose Phosphates in the Metabolic Pathway. Plant Physiol 87, 311-9 (1988).

57. Pichersky, E., Bernatzky, R., Tanksley, S. D. \& Cashmore, A. R. Evidence for selection as a mechanism in the concerted evolution of Lycopersicon esculentum (tomato) genes encoding the small subunit of ribulose-1,5-bisphosphate carboxylase/oxygenase. Proc Natl Acad Sci USA 83, 3880-4 (1986).

58. Schauber, C. et al. Rad23 links DNA repair to the ubiquitin/proteasome pathway. Nature 391, 715-8 (1998).

59. Ortolan, T. G. et al. The DNA repair protein rad23 is a negative regulator of multi-ubiquitin chain assembly. Nat Cell Biol 2, 601-8 (2000).

\section{Acknowledgements}

This project was funded by the National Key Research and Development Program (2016YFD0101802), the National Natural Science Foundation of China (31370031), Henan Major Science and Technology Projects (161100110500) and Henan Science and Technology Innovation Outstanding Youth Funding (174100510001) of China.

\section{Author Contributions}

F.C. designed the project. N.Z. and L.Z. performed highly sensitive immune affinity purification and high resolution LC-MS/MS. N.Z., L.Z., C.S., Q.T., G.L., Y.W., D.C. and F.C. performed date analysis. N.Z. and F.C. wrote the paper.

\section{Additional Information}

Supplementary information accompanies this paper at https://doi.org/10.1038/s41598-017-13992-y.

Competing Interests: The authors declare that they have no competing interests.

Publisher's note: Springer Nature remains neutral with regard to jurisdictional claims in published maps and institutional affiliations.

Open Access This article is licensed under a Creative Commons Attribution 4.0 International License, which permits use, sharing, adaptation, distribution and reproduction in any medium or format, as long as you give appropriate credit to the original author(s) and the source, provide a link to the Creative Commons license, and indicate if changes were made. The images or other third party material in this article are included in the article's Creative Commons license, unless indicated otherwise in a credit line to the material. If material is not included in the article's Creative Commons license and your intended use is not permitted by statutory regulation or exceeds the permitted use, you will need to obtain permission directly from the copyright holder. To view a copy of this license, visit http://creativecommons.org/licenses/by/4.0/.

(C) The Author(s) 2017 